Document downloaded from:

http://hdl.handle.net/10251/93324

This paper must be cited as:

Salvador Zuriaga, P.; Naranjo Ornedo, V.; Insa Franco, R.; Teixeira, P. (2016). Axlebox accelerations: Their acquisition and time frequency characterisation for railway track monitoring purposes. Measurement. 82:301-312. doi:10.1016/j.measurement.2016.01.012

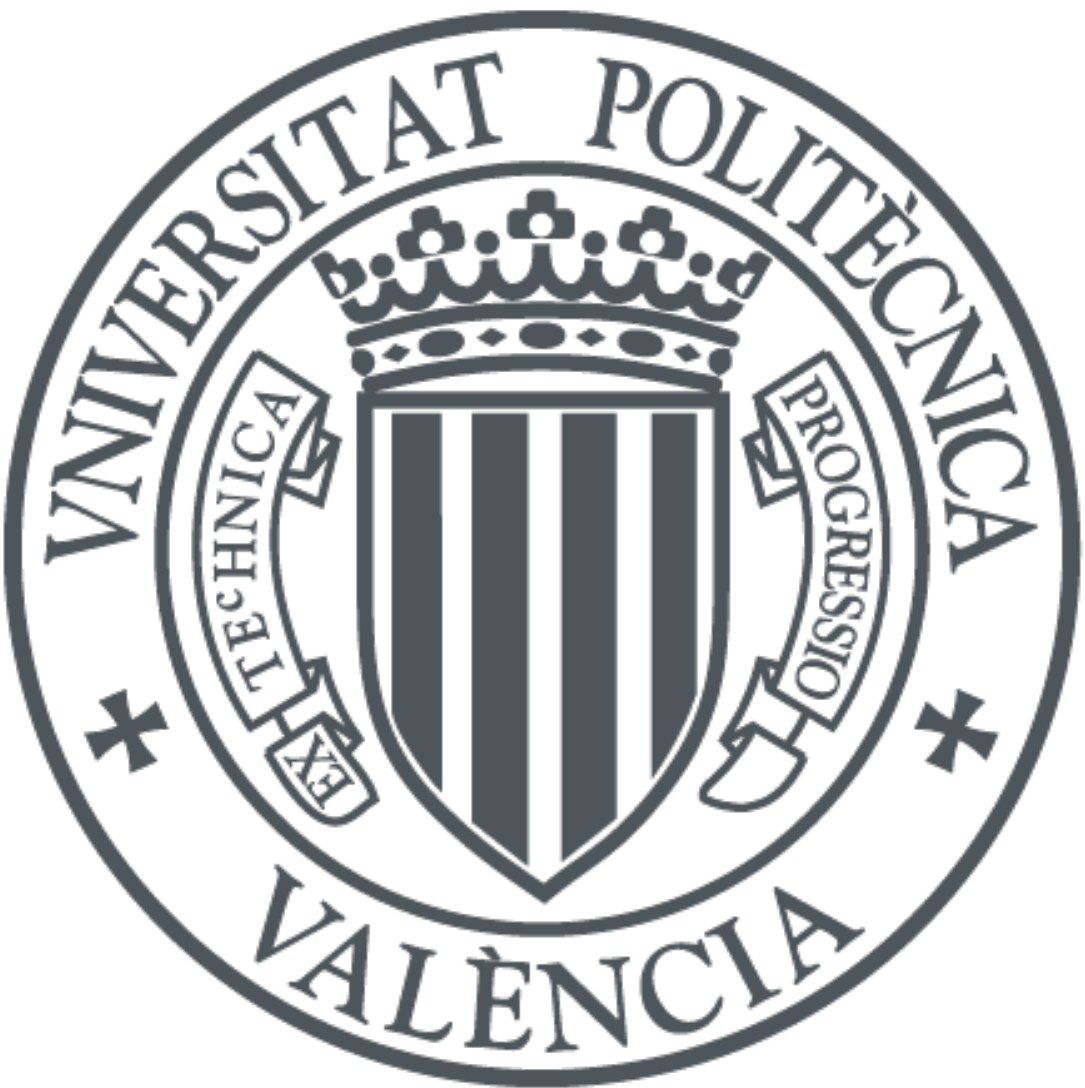

The final publication is available at

http://doi.org/10.1016/j.measurement.2016.01.012

Copyright Elsevier

Additional Information 


\title{
Axlebox accelerations: their acquisition and time-frequency characterization for railway track monitoring purposes
}

\author{
Pablo Salvador*, a ; Valery Naranjo ${ }^{\text {}}$; Ricardo Insa ${ }^{a}$; Paulo Teixeira ${ }^{c}$ \\ a Institute of Transport and Territory, Universidad Politécnica de Valencia. Camino de \\ Vera, s/n 46022 Valencia (Spain) \\ b Institute of Human Centred Technology LabHuman, Universidad Politécnica de \\ Valencia. Camino de Vera, s/n 46022 Valencia (Spain) \\ c CESUR/DECIVIL, Instituto Superior Técnico (IST), Technical University of Lisbon, \\ Av. Rovisco Pais 1049-001 Lisboa, Portugal.
}

*Corresponding author. E-mail: pabsalzu@cam.upv.es. Tel.: +34 648231661. Fax: +34 963877379

\begin{abstract}
Railway track maintenance is becoming a real challenge for Railway Engineers due to the need of meeting increasingly high quality requirements by means of cost-effective procedures. Frequently, this can be only achieved by implementing some technological developments from other fields into the railway sector, such as Digital Signal Processing. Indeed, the present work delves into data acquisition and processing techniques in order to enhance track surveying processes. For this purpose, run tests on the Metropolitan Rail Network of Valencia (Spain) were carried out, and axlebox accelerations were gathered and analysed in different ways. The results determined the optimal sampling and filtering frequencies as well as the location of accelerometers along the train. Furthermore, by means of spectral analysis and time-frequency representations, diverse track defects, track singularities and vibration modes can be clearly identified. It is shown how, with a Hamming time window of $0.5 \mathrm{~s}$ and an overlapping of 95\%, a wide set of track defects can be detected, without the need of complementary analyses. These values yield the best results as they are a good compromise between time and frequency resolution and allow for appropriate pattern recognition of the corresponding track singularities and resonant frequencies.
\end{abstract}

Keywords

Track maintenance; track surveying; axlebox accelerations; time-frequency representation; track spectrogram.

\section{Introduction}

On a panorama in which railway maintenance needs are increasing due to higher requirements, axlebox accelerations, i.e. accelerations being measured at the ends of the wheelsets, although currently being used for track surveying purposes, still offer a huge potential for obtaining more information about the tracks conditions.

Focusing on the track surveying part of the overall track maintenance process, there are different approaches for this purpose, with their respective advantages and drawbacks. Among these procedures, axlebox accelerometers have proved to be versatile enough to be introduced in different railway subsystems, e.g. High Speed or Metro trains, for 
detecting track irregularities of different wavelengths and occurrence. Therefore, they are one of the most promising methods for track surveying processes beyond its already widespread use [1-5] in comparison with other techniques and devices [6]. They present, though, the drawback of needing some maintenance and being quite delicate in a not friendly envirnoment [7]. They are also suitable for implementation in railway track degradation predictive models, as a useful indicator of the tracks maintenance conditions $[8,9]$.

The analysis of track conditions by means of wheelset, bogie frame and car body accelerations appears in the UIC (International Railway Union) leaflet number 518 [10] for the homologation of rolling stock and evaluation of both comfort and safety. Nevertheless, for the case of vehicles with bogies, only vertical and lateral car body and lateral bogie frame accelerations are measured. The use of vertical axlebox accelerations is not addressed. Other studies delve on condition monitoring of suspension bogies, mainly for analysing vehicle performance [11].

From the point of view of track maintenance, several authors have based their research on axlebox accelerations. They allow detecting and identifying some singular track defects such as squats [12-16], bolt tightness of fish-plated joints [17] and other short track defects $[18,19]$. They further serve as a basis for the calibration and validation of different wear prediction models [20]. Other works also applied axlebox accelerations for the detection and analysis of rail corrugation [2, 21-23], wheel/rail slippage [24] or for more general track surveying purposes, taking into account track defects of several wavelength ranges [25-28]. The use of accelerometers was furthermore applied to monitor the working conditions of turnouts. In this case, the accelerometers were not only placed at the wheelsets and the bogie frame, but also at the nose of the turnout frog [29].

In this sense, choosing the sampling frequency is not a trivial matter. In studies dedicated to short wavelength or singular track defects, sampling resolution can achieve high values, hence providing higher resolution and frequency content. In these cases, data may be recorded at a sampling frequency of $25.6 \mathrm{kHz}$ at speeds lower than $40 \mathrm{~km} / \mathrm{h}$ for tramway track monitoring purposes [17] or at speeds around $110 \mathrm{~km} / \mathrm{h}$ for conventional tracks [20]. Despite such a high sampling frequency value, in this last case only frequencies up to $2 \mathrm{kHz}$ are analysed. Furthermore, data may also be recorded every $2 \mathrm{~mm}$ at walking speeds [30].

On the contrary, for general inspection of railway lines, there must be a compromise between the resolution and the amount of recorded data. Sampling frequencies are thus lowered down to $2 \mathrm{kHz}$ [26] or $3 \mathrm{kHz}$ [25] for speeds up to $300 \mathrm{~km} / \mathrm{h}$. _The Nyquist theorem states that the maximum frequency which can be observed is half the sampling frequency. This presents the drawback that those track defects whose excitation frequency is higher than half of the sampling frequency are not detected. Nevertheless, for the case of Ref. [25], track defects longer than $5.6 \mathrm{~cm}$, which represent the major part, could be detected. For the case of Ref. [26], the lower operational speeds allow detecting the whole range of track defects.

However, as important as how to record axlebox accelerations is how to process and analyse them in order to extract the most information possible. Among other methods, the wavelet theory was applied for achieving a proper time-frequency characterisation 
of such accelerations $[13,17,25,31]$. Moreover, the wavelet theory was compared with the more conventional Fourier analysis, showing the advantages and drawbacks of each method [32]. Other works, specifically applied to rail corrugation detection, proposed solving the time-frequency uncertainty by means of the class of Auto-RegressiveMAtched Filterbank (AR-MAFI) methods [30] and by means of Polynomial Chaos Expansion and Point Estimate Method principles [33]. Finally, some other studies analyse railway tracks inspection based on the vibration response by means of the Hilbert-Huang transform [34].

The purpose of this paper is to improve current dynamic surveying approaches extensively applied in main lines and present an analysis tool capable of showing track properties and defects in a comprehensive way. It is shown how, by means of an appropriate tuning of the spectrogram defining parameters, track singularities and defects such as welded and glued joints, turnout frogs, squats and changes on track typologies are detected. Similarly, different track vibration modes, e.g. parametric excitation, p2 resonance due to rail corrugation, sleeper on ballast, rail on railpad and pin-pin resonance can be perceived in a single diagram. These track vibration modes extend from $10 \mathrm{~Hz}$ to $1 \mathrm{kHz}$ for the case of the analysed tracks of Metrovalencia (Valencia, Spain). In practical terms, this research pretends is to develop a tool for assisting engineers in charge of tracks supervision. This approach not only detects acceleration peaks caused by track defects, but also gives information regarding the nature of the defects and singularities with no further or parallel analysis. In this way, no further or parallel analysis is needed, thus simplifying track monitoring processes are simplified and avoiding extra visual inspections to the railway tracks are avoided.

Prior to this, some important questions, such as the most appropriate frequency band, the sampling frequency and the location of the accelerometers are addressed. Finally, this fine-tuned spectrogram is compared with other suitable time-frequency representation already employed in railway track analyses: the wavelet transform [12, $13,25,28,32]$.

\section{Train/track dynamic interaction and classification of track defects}

\subsection{Train/track dynamic interaction}

A train running on a track can be modelled as a system of masses, springs and dampers. Fig. 1 shows the different parts trains and tracks consist of and how they can be conceptually modelled. From this figure, it can be seen that any significant alteration in any of the elements will affect the rest of the system. For example, a single defect located on the rail surface would excite the different masses not only in the train, but also and in the track itself; and the same would happen with a stiffness variation in the railpads or in the ballast, a loose sleeper, etc. For this reason, axlebox accelerations are a useful indicator of track maintenance conditions.

In this way, a train may be conceptually modelled as a three masses system, i.e. the car body, the bogie frame and the wheelset. Each mass is connected to its adjacent by means of the secondary and primary suspensions, respectively. Similarly, a track may be modelled as a two masses system, i.e. the rail and the sleeper. Elasticity between the rail and the sleeper is procured by the railpads, whereas the elasticity between the 
sleepers and the ground is provided by the ballast. Interaction between train and track happens in the wheel/rail contact, a phenomenon for which there is a fully developed theory [35, 36]. Conceptually, wheel/rail contact may be modelled as a very stiff spring, compared to the rest of the elements.

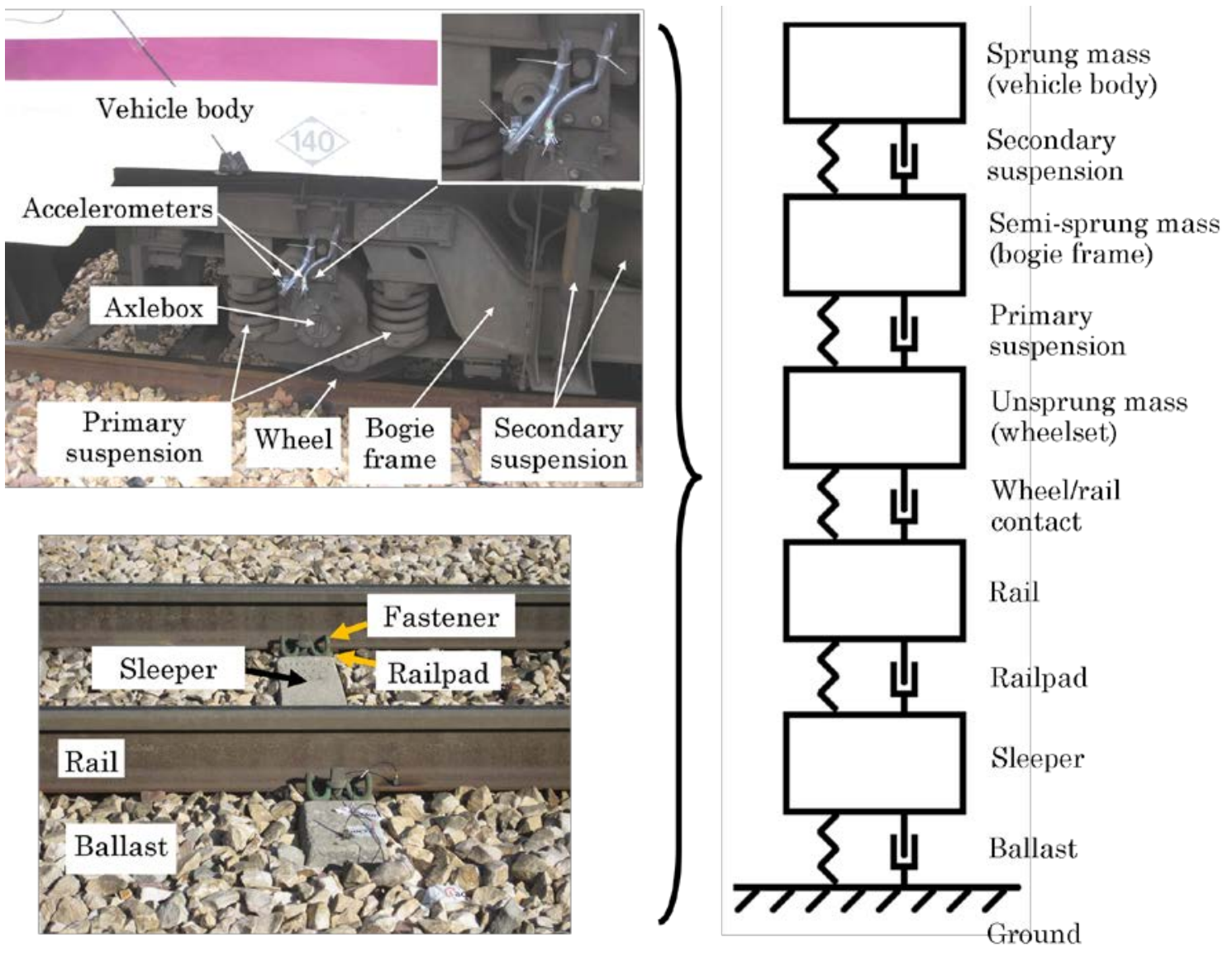

Fig. 1. Main train and track components, their representation in a conceptual train/track dynamic interaction model and location of accelerometers

\subsection{Classification of track defects}

Since track defects of different wavelengths are repaired by means of different techniques, their classification depending on their wavelength becomes a suitable criterion. Two main groups of defects can be identified at first: those coming from the loss of track vertical geometry, whose wavelengths are usually longer than $2 \mathrm{~m}$; and those related to rail corrugation or isolated rail defects (e.g. squats, spalling) in which a loss of material is produced at the top of the rails heads while the track keeps its vertical alignment. Table 1 shows the classification of vertical track defects used by Spanish Railway Administrator Adif, which is taken as a reference in this work.

\begin{tabular}{|l|l|c|l|}
\hline Type of defect & Classification & $\begin{array}{l}\text { Wavelength } \\
\text { range (m) }\end{array}$ & Examples of defects \\
\hline & Very short & $0.03-0.06$ & $\begin{array}{l}\text { Rail joints, very short } \\
\text { wavelength rail corrugation, } \\
\text { small squats }\end{array}$ \\
\cline { 2 - 4 } & Short & $0.06-0.25$ & Short wavelength rail \\
\hline
\end{tabular}




\begin{tabular}{|l|l|c|l|}
\hline & & & $\begin{array}{l}\text { corrugation, medium size } \\
\text { squats }\end{array}$ \\
\cline { 2 - 4 } & Medium & $0.25-0.60$ & $\begin{array}{l}\text { Medium wavelength rail } \\
\text { corrugation, large squats, } \\
\text { turnout frogs }\end{array}$ \\
\cline { 2 - 4 } & Long & $0.60-2$ & $\begin{array}{l}\text { Long wavelength rail } \\
\text { corrugation, ballast fouling }\end{array}$ \\
\hline & Short & $2-25$ & $\begin{array}{l}\text { Changes on track vertical } \\
\text { stiffness }\end{array}$ \\
\cline { 2 - 4 } & Medium & $25-70$ & $\begin{array}{l}\text { Medium wavelength vertical } \\
\text { misalignment }\end{array}$ \\
\cline { 2 - 4 } & Long & $70-120$ & $\begin{array}{l}\text { Long wavelength vertical } \\
\text { misalignment }\end{array}$ \\
\hline
\end{tabular}

Table 1. Classification of track vertical defects upon their wavelengths. Source: Adif (internal document)

In this way, when a train runs along a track with a defect of a given wavelength $\lambda$ at a certain speed $V$, it experiments a vertical movement whose frequency is given by

$$
f=V / \lambda \text {. }
$$

Considering the rolling stock as a multibody dynamic system, it consists of some unsprung masses, located below the primary suspension and whose natural frequencies usually range between 20 and $1000 \mathrm{~Hz}$; semi-sprung masses, above the primary suspension with natural frequencies usually from 5 to $20 \mathrm{~Hz}$; and sprung masses, located above the secondary suspension, with natural frequencies usually ranging from $0.7 \mathrm{~Hz}$ to $5 \mathrm{~Hz}$. Furthermore, the track elements usually have their resonant frequencies between 30 and $2000 \mathrm{~Hz}$ [37]. Only small excitations due to the railhead roughness and very short railhead corrugation fall beyond $3000 \mathrm{~Hz}$ as pointed out in [6].

\begin{tabular}{|c|c|c|c|c|c|c|c|c|}
\hline & \multicolumn{8}{|c|}{ wavelength (m) } \\
\hline & 0.03 & 0.06 & 0.25 & 0.6 & 2 & 25 & 70 & 120 \\
\hline 300 & 2778 & 1389 & 333 & 139 & 42 & 3.3 & 1.2 & 0.7 \\
\hline 250 & 2315 & 1157 & 278 & 116 & 35 & 2.8 & 1.0 & 0.6 \\
\hline 220 & 2037 & 1019 & 244 & 102 & 31 & 2.4 & 0.9 & 0.5 \\
\hline 200 & 1852 & 926 & 222 & 93 & 28 & 2.2 & 0.8 & 0.5 \\
\hline 160 & 1481 & 741 & 178 & 74 & 22 & 1.8 & 0.6 & 0.4 \\
\hline 120 & 1111 & 556 & 133 & 56 & 17 & 1.3 & 0.5 & 0.3 \\
\hline 100 & 926 & 463 & 111 & 46 & 14 & 1.1 & 0.4 & 0.2 \\
\hline 80 & 741 & 370 & 89 & 37 & 11 & 0.9 & 0.3 & 0.2 \\
\hline 60 & 556 & 278 & 67 & 28 & 8 & 0.7 & 0.2 & 0.1 \\
\hline 50 & 463 & 231 & 56 & 23 & 7 & 0.6 & 0.2 & 0.1 \\
\hline
\end{tabular}

Table 2. Excitation frequencies of different wavelengths when travelling at different speeds and its possible coupling to the different train and track elements: $\square$ Track elements; $\square$ Unsprung masses; $\square$ Semi-sprung masses; $\square$ Sprung masses 
Railway Engineers who are responsible of the tracks' maintenance must avoid these track defects as they produce a resonance effect in the train or the tracks. This effect amplifies the train movements and increases dynamic loads and material wear [38]. This also leads to some discomfort events and derailment risks. In this sense, Table 2 shows the frequencies excited by the boundary wavelengths of Table 1 for different speeds and their possible coupling with the natural frequencies of the aforementioned train masses and track elements.

As commented previously in Section 1, in order to properly detect the different track defects and singularities, the sampling frequency must be, at least, twice the frequency yielded by Table 2 for a given wavelength at a certain speed. For the case of a High Speed Train traveling at $300 \mathrm{~km} / \mathrm{h}$, if short track defects of $3 \mathrm{~cm}$ are to be detected, the sampling frequency ought to be at least $5556 \mathrm{~Hz}$. For the case of the present paper, where there is a maximum operational speed of $80 \mathrm{~km} / \mathrm{h}$, the sampling frequency must be at least $1482 \mathrm{~Hz}$.

\section{Experimental setup}

In order to analyse all these issues, the following experiment was set up: two accelerometers measuring vertical accelerations where placed at the first trailer wheelset and at the first motor wheelset of the train, respectively. The reason of these locations is the fact that the first wheelsets of each coach, and especially the first wheelset of the complete train unit, absorb the major part of dynamic loads. Furthermore, the difference between a motor bogie and a trailer bogie may let see any possible difference in the recorded signals caused by some vibration sources such as motors, gears or induced currents. Table 3 shows the accelerometers main features.

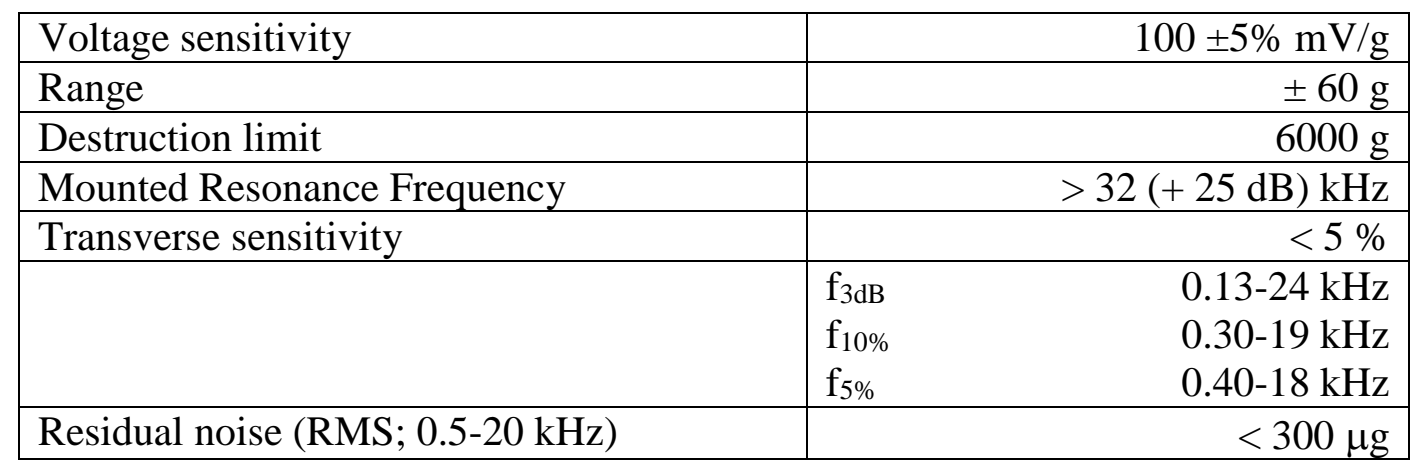

Table 3. Main features of accelerometers KS76C100 manufactured by MMF

Run tests were performed in two stretches of line 1 of Metrovalencia. In the first place, several runs took place between the stations of Paterna and Fuente del Jarro in a 2.35 $\mathrm{km}$ single ballasted track. This allowed testing different sampling frequencies and lowpass filters as well as proving the track surveys' repeatability. Secondly, the stretch between Valencia Sud and Paterna and vice-versa was monitored too, which consists on a $12.20 \mathrm{~km}$ double track of similar features as in the previous stretch. This allowed surveying a wider number of track defects of different type and magnitude.

Metrovalencia operates the commuter services in the metropolitan area of Valencia (Spain) connected by a narrow gauge railway network, i.e. 1,000 mm. The track 
configurations are: ballasted track with single-block concrete sleepers, and Stedef slab track, used only in some stations and sharp curves. The main features of both systems are shown in Table 4.

The train used in the tests was an Electrical Multiple Unit (EMU) of the 4300 series, which has 4 cars of two bogies each one, being motorised all four wheelsets of the first and the last cars. Its maximum speed is $80 \mathrm{~km} / \mathrm{h}$. Table 5 shows the different runs with the specific sampling frequency and anti-aliasing filtering choice they were carried out with. Fig. 1 shows the experimental setup.

\begin{tabular}{|l|l|l|}
\hline & Ballast & Slab \\
\hline Rails & UIC-54 & UIC-54 \\
\hline Rail mass per unit length, $m$ & $54 \mathrm{~kg} / \mathrm{m}$ & $54 \mathrm{~kg} / \mathrm{m}$ \\
\hline Rail elasticity modulus, $E$ & $210 \cdot 10^{9} \mathrm{~N} / \mathrm{m}^{2}$ & $210 \cdot 10^{9} \mathrm{~N} / \mathrm{m}^{2}$ \\
\hline Rail moment of inertia $I$ & $2314 \cdot 10^{-8} \mathrm{~m}^{4}$ & $2314 \cdot 10^{-8} \mathrm{~m}^{4}$ \\
\hline Railpad stiffness, $k_{\mathrm{p}}$ & $1.2 \cdot 10^{8} \mathrm{~N} / \mathrm{m}$ & $1.2 \cdot 10^{8} \mathrm{~N} / \mathrm{m}$ \\
\hline Mass of half a sleeper, $M$ & $90 \mathrm{~kg}$ & $90 \mathrm{~kg}$ \\
\hline Distance between sleepers, $d$ & $0.7-0.8 \mathrm{~m}$ & $0.9 \mathrm{~m}$ \\
\hline Stiffness under the sleepers, $k_{\mathrm{b}}$ & $2.6 \cdot 10^{8} \mathrm{~N} / \mathrm{m}$ & $2.6 \cdot 10^{8} \mathrm{~N} / \mathrm{m}$ \\
\hline
\end{tabular}

Table 4. Different features for both track configurations.

\begin{tabular}{|l|l|}
\hline $\begin{array}{l}\text { Run test } \\
\text { number }\end{array}$ & $\begin{array}{l}\text { Sampling frequency/cut-off } \\
\text { frequency }(\mathrm{Hz})\end{array}$ \\
\hline 1 & $2,500 / 1,000$ \\
\hline 2 & $15,000 / 5,000$ \\
\hline 3 & $15,000 / 5,000$ \\
\hline 4 & $2,500 / 1,000$ \\
\hline 5 & $2,500 / 1,000$ \\
\hline
\end{tabular}

Table 5. Specific features of the different run tests showing the sampling frequency and the anti-aliasing filtering choice.

\section{Description of data collection and processing}

\subsection{Data collection}

Taking a maximum speed of $80 \mathrm{~km} / \mathrm{h}$ and a minimum rail wavelength of $2.5 \mathrm{~cm}$ (i.e. the shortest possible wavelength of rail corrugation to be analysed) Eq. (1) yields a maximum frequency of $890 \mathrm{~Hz}$. For this reason, an anti-aliasing analogical filter of 1 $\mathrm{kHz}$ has been chosen. Because of the Nyquist theorem, the sampling frequency has been set to $2.5 \mathrm{kHz}$. In order to show that no relevant frequencies are dismissed during the digitalisation process, the anti-aliasing filter has been raised up to $5 \mathrm{kHz}$ in two of the five runs, with a sampling frequency of $15 \mathrm{kHz}$. Now let us denote both methods as 2.5/1 and 15/5, respectively. 


\subsection{Data processing}

The data collected has been analysed by means of a time-frequency representation based on the spectrogram. The spectrogram takes firstly the digitalised signal and splits it in shorter samples of a given window length $L$ with a given overlap $P$ between one sample and the next one. After this, the Short Time Fourier Transform (STFT) is calculated for each sample. In the graphic representation, the $x$-axis corresponds to the time domain, whereas the $y$-axis stands up for the frequency domain. In this way, each STFT is represented column-wise at the instant corresponding with the mid-time of the sample. The amplitudes of the different frequencies are represented with a colour scale, obtaining a $2.5 \mathrm{D}$ representation.

STFT is performed in the following way:

$X(k)=\sum_{n=0}^{N-1} x(n) \omega_{N}^{n k}$

where

$X(k)$ represents samples of the signal in the frequency domain with $k=0,1, \ldots, N-1$ in the normalised frequencies $f_{\mathrm{k}}=k / N . k=0, \ldots, N-1$.

$x(n)=$ signal samples in the time domain

$N=$ number of points the STFT is calculated with

$\omega_{N}=\mathrm{e}^{(-2 \pi \mathrm{i}) / N}$

Amplitudes are represented in logarithmic scale from the Power Spectral Density (PSD) of the signal. If $A(r, c)$ denotes the amplitude at the instant $t_{r}$ for the frequency $f_{c}$, then the PSD at the same point $(r, c)$ is given by

$P(r, c)=a|A(r, c)|^{2}$

with

$a=\frac{2}{F_{s} \sum_{n=1}^{l}|w(n)|^{2}}$

where $F_{\mathrm{s}}$ is the sampling frequency and $w(n)$ denotes the window function. Notice that, at 0 and the Nyquist frequencies, the factor of 2 in the numerator is replaced by 1 .

In this way, the amplitude in the spectrogram, in which the colours scale is based on, becomes:

$S(r, c)=10 \log _{10} P(r, c)$

The overlap $P$ has been set as a fixed percentage of $L$, around $95 \%$. The number of points the Fourier Transform is calculated with, $N$, is set by observing that it must be a power of 2 and must be larger than 4 times the window length, i.e.

$$
N=2^{s}>4 l
$$

where $s \in \mathbb{N}$, so as to achieve sufficient frequency interpolation [39].

\subsection{Calibration of time window length}

Let us pay attention to the window length. As it is known in the time-frequency representation theory, the higher accuracy is achieved in one variable, the lower accuracy is achieved in the other. By setting wide time windows, frequency resolution increases, but the time resolution keeps rather low, i.e. track defects cannot be properly located. On the contrary, narrow time windows allow a better time resolution, but the signal frequency content in that time is distorted. Therefore, the window length must become a compromise between both time and frequency resolutions. 
The window length has been calibrated from two different criteria: time and frequency. For the time criterion, it has been started with $2 \mathrm{~s}$ width time windows and they have progressively been narrowed until no additional substantial information in the time domain was obtained. For the frequency criterion, it has been started with $0.1 \mathrm{~s}$ width time windows and they have progressively been widened until no additional substantial information in the frequency domain was obtained. Both criteria give an upper and a lower window width which become the range of most suitable window lengths. This calibration has been carried out for two different time windows: rectangular and Hamming.

Table 6 shows the different results for each tested window length. Track singularities and vibration modes are described in section 5.3 as they are identified in the spectrograms. The result is that window lengths between $0.5 \mathrm{~s}$ and 0.3 seconds give the best appearances.

Now, the number of windows necessary to cover a certain time series is given by:

$w=\frac{T f_{S}-L}{L-P}$

where $w$ is the number of windows, $T$ is the total length of the time series, $f_{\mathrm{s}}$ is the sampling frequency and $P$ is the overlap between windows. As can be seen, the number of necessary windows increases as the windows length shortens. Therefore, a window length of $0.5 \mathrm{~s}$ is chosen since a lower number of windows and hence less computational effort is needed.

Regarding the time windows, it is seen that Hamming windows achieve similar results than rectangular windows of $2 / 3$ of its length. For the same reason of computational efficiency, Hamming windows have been chosen.

\begin{tabular}{|l|l|}
\hline Window length & Observations \\
\hline $2 \mathrm{~s}$ (5000 samples) & Track singularities such as rail joints closer than 20 m overlap \\
\hline $1 \mathrm{~s}$ (2500 samples) & $\begin{array}{l}\text { Track singularities closer than 10 m overlap. Differences } \\
\text { between turnout frogs and glued joints cannot be appreciated }\end{array}$ \\
\hline 0.5 s (1250 samples) & Track singularities are properly identified \\
\hline $0.1 \mathrm{~s}$ (250 samples) & Parametric excitation and vibration of sleeper on ballast overlap \\
\hline 0.2 s (500 samples) & $\begin{array}{l}\text { Parametric excitation and vibration of sleeper on ballast still } \\
\text { overlap but start to be distinguished }\end{array}$ \\
\hline 0.3 s (750 samples) & $\begin{array}{l}\text { Parametric excitation and vibration of sleeper on ballast can be } \\
\text { mostly distinguished }\end{array}$ \\
\hline
\end{tabular}

Table 6. Observed appearances for different window lengths

\section{Results and discussion}

\subsection{Sampling frequency and anti-aliasing filter}

Figure 2 shows the spectrogram of a track fragment between Paterna and Fuente del Jarro with data registered at $15 / 5 \mathrm{kHz}$ (a) and the same data after being digitally filtered with a Butterworth filter of 4 poles and sub-sampled down to $2.5 \mathrm{kHz}$ (b) The train speed is variable between 75 and $30 \mathrm{~km} / \mathrm{h}$. There can be seen that no relevant frequency 
content is found beyond $1000 \mathrm{~Hz}$. There are only some "vertical lines", which correspond to the wheel rolling over the welded and glued joints and a "horizontal line" around $2000 \mathrm{~Hz}$ which can be associated to one of the vibration modes of the wheelset. Only the excitation caused by the glued joint at $t=174 \mathrm{~s}$ extends up to $6.5 \mathrm{kHz}$, but it can be clearly distinguished in the $0-1000 \mathrm{~Hz}$ frequency band. Track singularities are further discussed in section 5.3. This shows evidence that data registration at $2.5 / 1 \mathrm{kHz}$ provides sufficient information for detecting such "impacts" at the rail joints, since the resonant frequency at $2000 \mathrm{~Hz}$ does not correspond to any track defect. Therefore, subsequent analyses are only shown for $2.5 / 1 \mathrm{kHz}$. For other railway systems, though, a higher frequency band may be required.
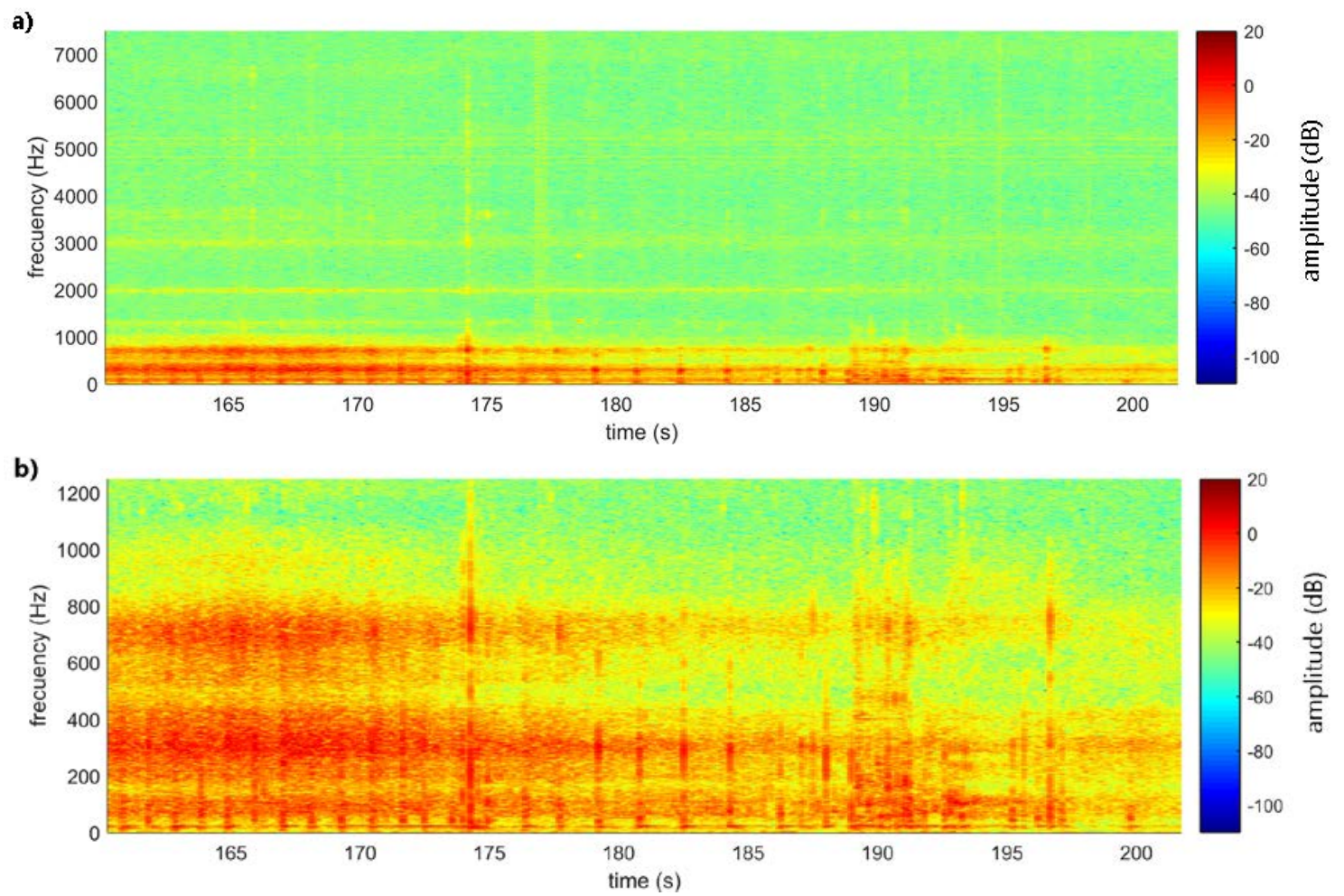

Fig. 2. Spectrogram showing the same accelerations sampled at $15 \mathrm{kHz}$ (a) and at 2.5 $\mathrm{kHz}$ (b). Speed $75-30 \mathrm{~km} / \mathrm{h}$.

\subsection{Location of axlebox accelerometers}

As commented in section 3, accelerometers have been placed at two different locations: the axlebox of a motor wheelset and the axlebox of a trailer wheelset. Figure 3a shows the spectrogram for the motor wheelset and so does Fig. $3 \mathrm{~b}$ for the trailer wheelset. Both spectrograms correspond to the same track stretch and were recorded in the same run, although there is a slight shift of approximately $1.5 \mathrm{~s}$ because of the distance between both wheelsets. The train speed varies between 10 and $30 \mathrm{~km} / \mathrm{h}$ as it was speeding up.

Figure 3a shows some horizontal and diagonal parallel lines at different frequencies. Such frequencies are caused by the vibrations produced by the different current harmonics induced in the motor mounted on the bogie. Since such vibrations appear throughout the frequency band, they may hide the actual track defects, thus hindering the analysis of the track. It can be seen in figure 3b how these induced vibrations do not exist, resulting in a cleaner spectrogram. 

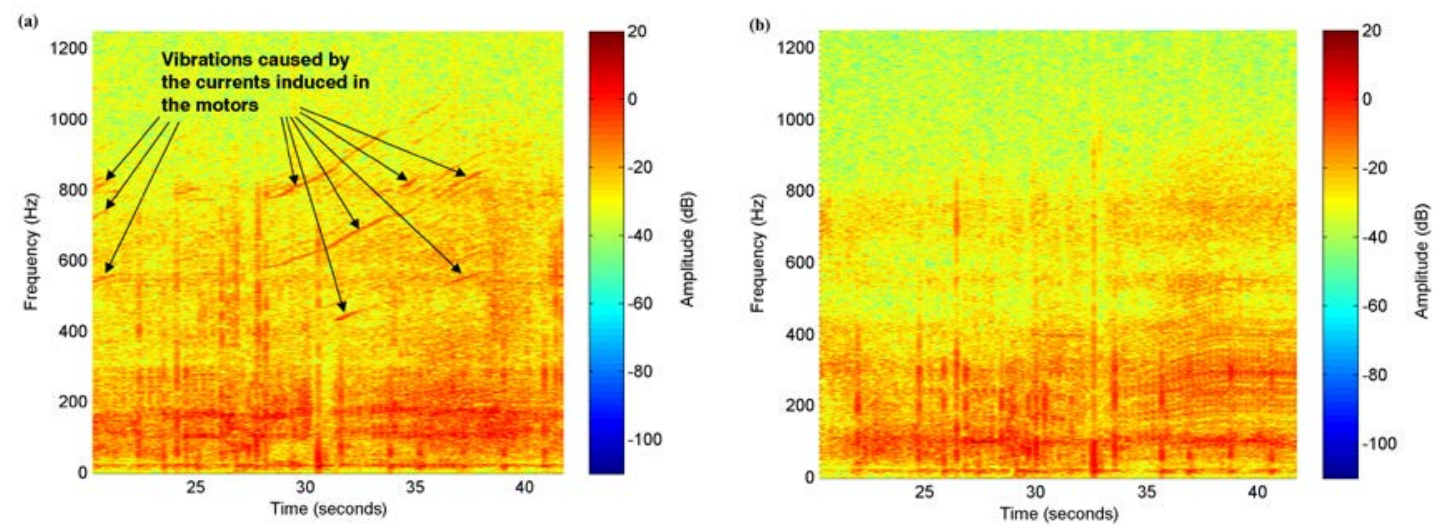

Fig. 3. Spectrogram corresponding to the same track stretch surveyed from a motor wheelset (a) and from a trailer wheelset (b). Speed $10-30 \mathrm{~km} / \mathrm{h}$

In contrast, the magnitude of the different track defects is the same in both pictures. Therefore it is more suitable, from the point of view of track surveying, to place the axlebox accelerators on the trailer wheelsets. Placing them at the motor wheelsets may have the advantage, though, that some information on the traction and electric braking efforts may be obtained, but this issue remains out of the scope of the present paper. On the following, only spectrograms from the trailer wheelset are shown.

\subsection{Observation of track defects}

By observing the spectrograms, two different groups of track defects can be easily distinguished: those which are isolated and those which appear along a certain track length. The first group may include not only track defects such as squats or rail spalling, but also track singularities such as welded joints, glued joints, turnouts or diamond crossings. Depending on the amplitude of the accelerations they cause, they may be considered as track defects too. The second group mainly includes vibration modes associated to different track configurations (e.g. ballast vs. slab), track vertical misalignment and rail corrugation. All the following representations have been obtained with a Hamming time window with $L=1250$, i.e. $0.5 \mathrm{~s}, P=1000$ and $N=2^{13}=8192$, as explained in section 4.2 .

The first group presents a column-wise appearance in the spectrogram, since they produce a short time duration impact on the wheel, thus exciting a wide range of frequencies. On the contrary, the second group appears as a horizontal band covering specific frequency ranges. On the following, the different track defects and singularities found in the spectrograms of the analysed track are described:

- Welded joints (Fig. 4, Fig 5): they are clearly defined in the range 0-400 Hz and have a width of $0.2 \mathrm{~s}$. Since Metrovalencia tracks use $18 \mathrm{~m}$ long rail bars, such joints appear periodically with a separation varying with the train speed.

- Glued joints (Fig. 4, Fig 6): their appearance in the spectrogram is featured by a vertical line covering a range of frequencies up to $800-900 \mathrm{~Hz}$ and a width of 0.2-0.3 s depending on their preservation conditions.

- Severe squats (Fig. 4, Fig. 7): they clearly cover the full range of the analysed frequencies ( 0 to $1250 \mathrm{~Hz}$ ) and its width has values of 0.2-0.4 s depending on its spread on the rail surface.

- Turnout frogs (Fig. 4, Fig. 8): they have their largest amplitudes in the frequency bands of $0-400 \mathrm{~Hz}$ and $550-800 \mathrm{~Hz}$. Their width at the bottom, i.e. $50-100 \mathrm{~Hz}$, is 
about 1-1.5 s depending on their maintenance conditions and it narrows as the frequency increases.

After the analysis of the recorded accelerations and the identification of potential track defects and singularities, visual inspections took place in those track points where some signal disturbances were found. As a matter of fact, figure 7 shows the squat at the turnout frog which appears in the spectrogram of fig. 4. At this respect, all the singularities found in the analysis were checked by means of visual inspections, with satisfactory results

Medium and severe squats are not likely to appear in metropolitan railway tracks with such light axle loads. As a matter of fact, the squat shown in the figure was the only one detected with a remarkable size in more than $14 \mathrm{~km}$ of analysed tracks. Upon the people in charge of the line's maintenance, it was probably caused by a ballast stone purposely put on the nose of the frog, rather than by fatigue due to the passing of the railway loads. The lack of a large variety of squats prevents us from delving in this issue. This squat was shown because the pattern it threw was quite noteworthy. The size of the squat was about 7-8 cm long, compared with the smaller squats from [15] and [16] with a size of $15 \mathrm{~mm}$. This produces the excited frequencies to be lower than in the research from these references, being extended up to $2.5 \mathrm{kHz}$.

Let us focus now on the three turnouts shown in Fig. 4. Whereas the first two were installed more recently and present almost no wear, the one at $t=885 \mathrm{~s}$ was installed in 1988 and presents a worn rail profile, which is reflected in the time bandwidth around $100 \mathrm{~Hz}$, widen up to $1.5 \mathrm{~s}$ and between 200 and $400 \mathrm{~Hz}$, widen up to $1 \mathrm{~s}$. Nevertheless, the range of excited frequencies remains invariable. Hence, the straight vertical line in the first turnout, covering the whole frequency range, is due to the presence of the squat.

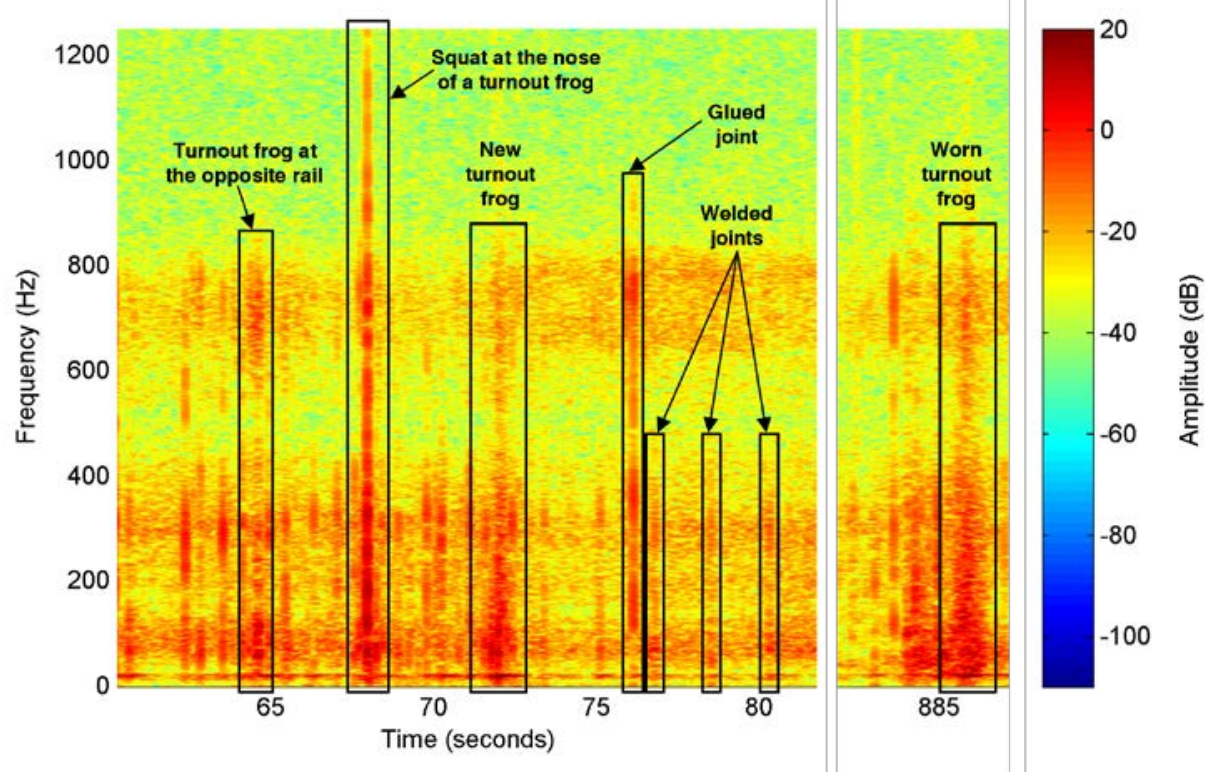

Fig. 4. Spectrogram showing different track singularities. Speed $35 \mathrm{~km} / \mathrm{h}$ 


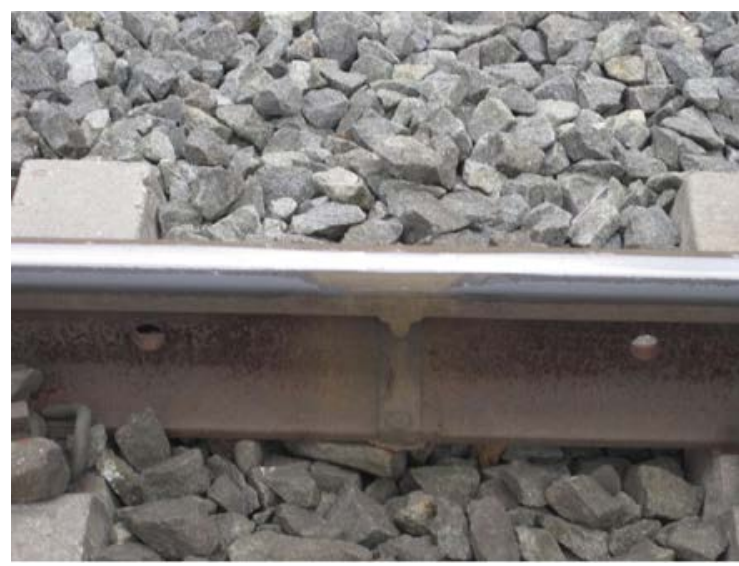

Fig. 5. Welded joint

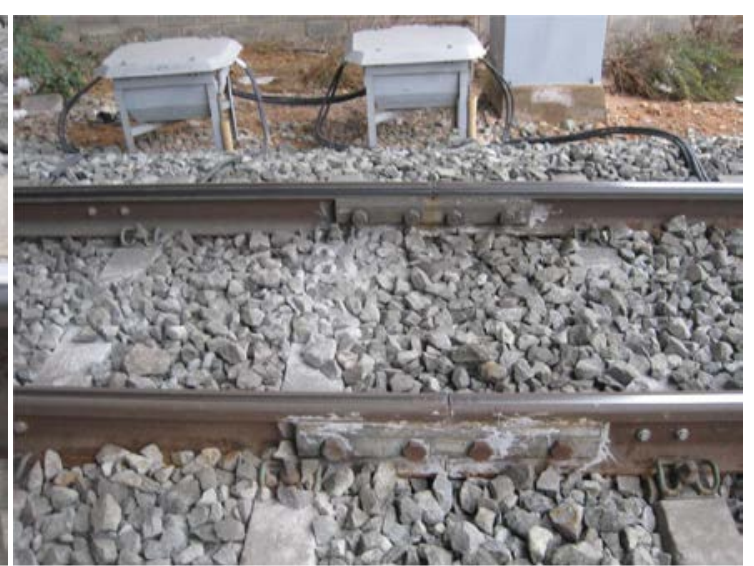

Fig. 6. Glued joint

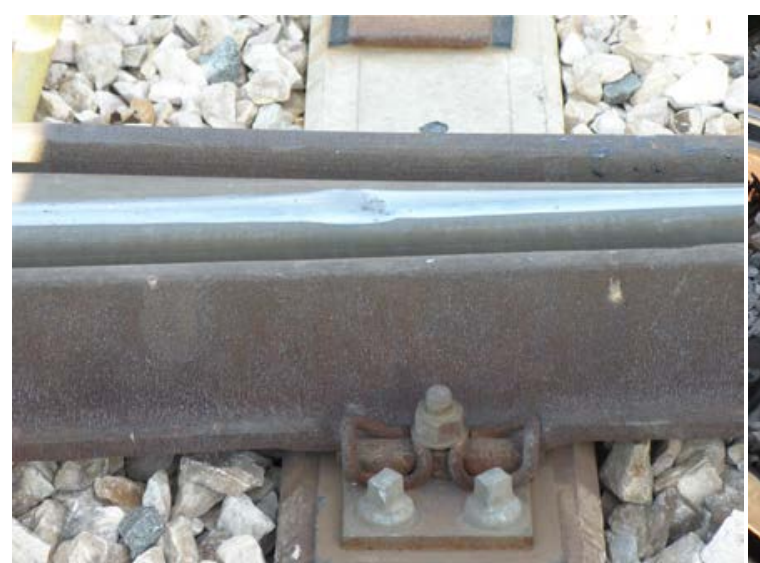

Fig. 7. Squat at the nose of a turnout frog

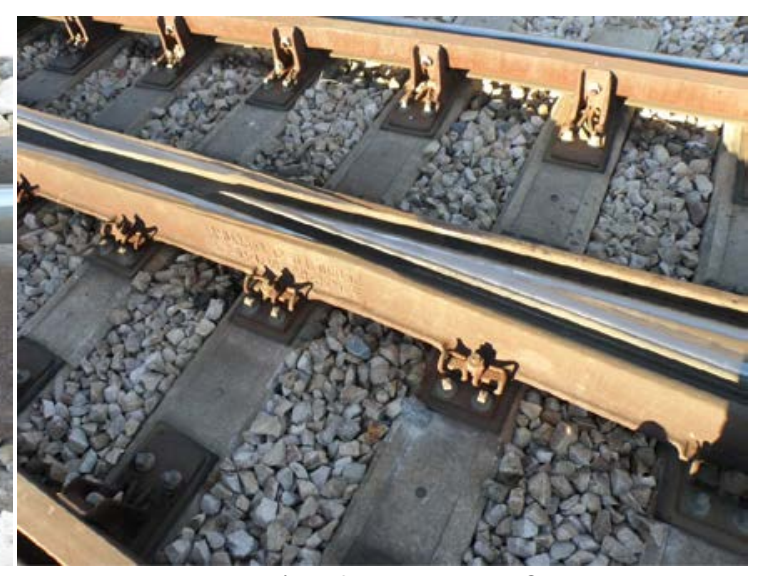

Fig. 8. Turnout frog

In addition, vibration modes which extend along a certain time period and therefore have a row-wise appearance in the spectrogram are:

- Parametric excitation $f_{\text {pe }}$ (Fig. 9): it is produced by the passing of a wheelset along the spans existing between the sleepers, describing thus a sinusoid whose wavelength is such distance, i.e. $70-80 \mathrm{~cm}$ for the case of Metrovalencia. It is reflected in the spectrogram as a thin horizontal line, whose frequency varies with the train speed but does not trespass the $30 \mathrm{~Hz}$.

- Vibration of sleeper on ballast $f_{\mathrm{s}}$ (Fig. 9): this is the resonant mode of the sleeper vibrating on the ballast, and is reflected as a thin horizontal line with a constant frequency around $20 \mathrm{~Hz}$.

- $\quad \mathrm{P} 2$ resonance $f_{\mathrm{P} 2}$ (Fig. 10): this is the resonant frequency of the unsprung mass of the bogie vibrating together with the rails, and it is reflected in the $50-100 \mathrm{~Hz}$ frequency band, as stated in [2, 40]. Since this resonant mode becomes a fixing mechanism of rail corrugation, its growth is detected in the colour scale as it happens in a stretch between the stations of Patraix and Safranar (Fig. 10).

- Vibration of rails on railpads $f_{\mathrm{r}}$ (Fig. 10): this corresponds with the resonant mode of the rails vibrating on the railpads, and is reflected in the spectrogram in the 200-400 $\mathrm{Hz}$ frequency band, achieving the highest amplitudes around 300 $\mathrm{Hz}$.

- Pin-pin resonance $f_{\mathrm{pp}}$ (Fig. 10): this corresponds with the first vibration mode of the rails considering the points where they are fastened to the sleepers as the nodes. It appears in the bandwidth of 650 to $800 \mathrm{~Hz}$ and the width is due to the 
sleepers being not separated an exact distance, but in the previously commented range.

These resonant frequencies are in accordance with those stated by the formulas in [37] and coincide with some experimental measurements carried out on the track elements [2].

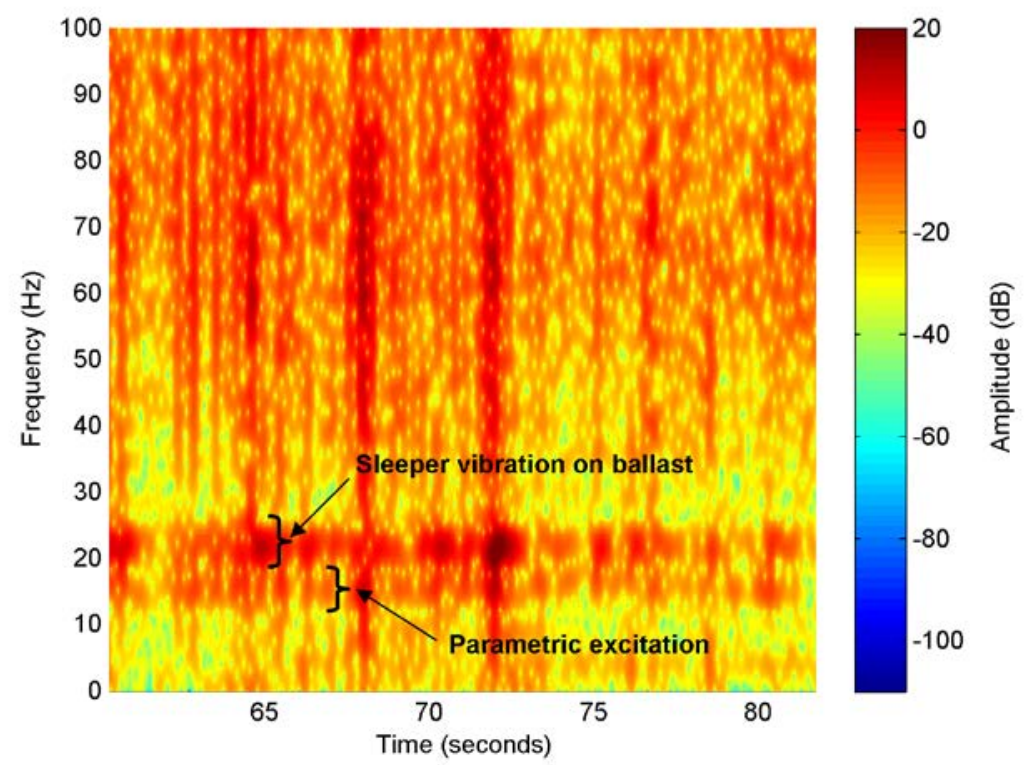

Fig. 9. Zoom to the 0-100 Hz frequency band for the same spectrogram shown in Fig. 4.

This means that any change in the track properties will affect not only these frequencies, but also $f_{\mathrm{P} 2}$. The detection of such changes is of relevance since they imply an increase of dynamic forces in these zones and the subsequent track degradation [41-43]. Indeed, the following changes can be perceived:

- Railway crossing (Fig. 10): in railway crossings, rails are confined in order to allow road vehicles to go across the tracks and they are fastened to wooden sleepers in order to fasten the check rails too. These changes wide the pin-pin frequency bandwidth from 650 to $850 \mathrm{~Hz}$ together with an increase of the amplitudes, as it is reflected in the spectrogram.

- Change from ballasted to slab track and vice-versa (Fig. 10): this change on slab track stretches increases up to $90 \mathrm{~cm}$. Thus, $f_{\mathrm{P} 2}$ and $f_{\mathrm{pp}}$ are lowered to the 30-50 $\mathrm{Hz}$ and $600-750 \mathrm{~Hz}$ frequency bands, respectively.
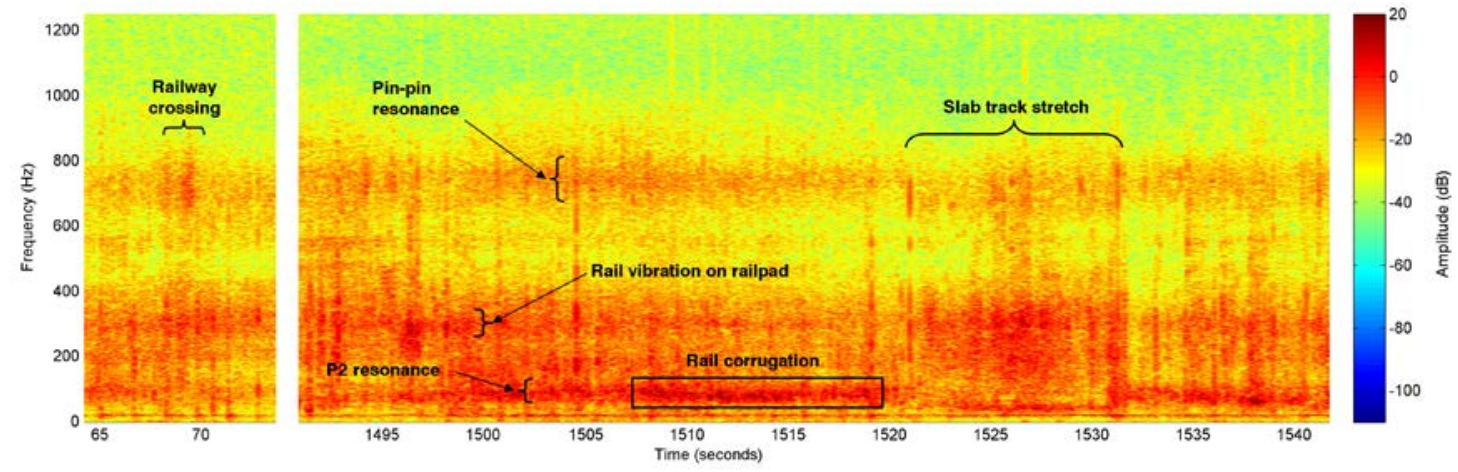
Fig. 10. Spectrogram showing different resonances, vibration modes and track configurations. Speed $40 \mathrm{~km} / \mathrm{h}$

\section{Comparison with wavelet transform}

As said before, several authors have based their research on time-frequency characterisation of railway track defects by using the wavelet transform (WT) [12, 13, $25,28,32]$. One of the most relevant WT properties is that the window length is not a fixed value, but varies along with the scale. Indeed, large scale wavelets, corresponding to the lower frequencies, have wide windows associated; and the opposite happens with small scale wavelets. This is viewed as an advantage respect to the conventional STFT by WT users. Nevertheless, the fact that window length varies with the scale parameter may produce a loss in frequency resolution for high frequencies and a loss in time resolution for low frequencies. This may result in some high frequency vibration modes, such as the pin-pin resonance, not being able to be properly identified.

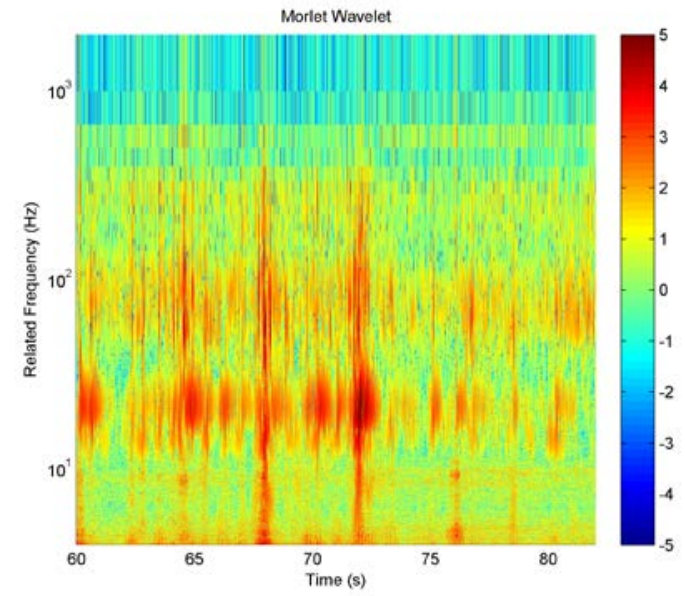

Fig. 11. Wavelet transform corresponding to the spectrogram of Fig. 4

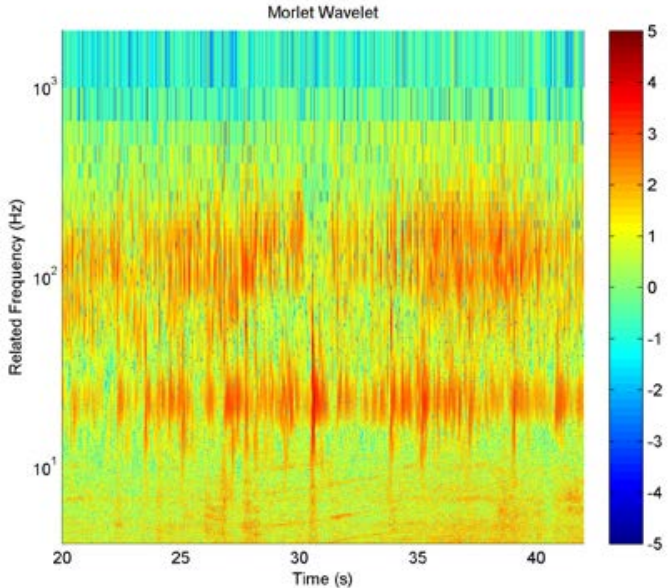

Fig. 12. Wavelet transform corresponding to the spectrogram of Fig. 3a.

Furthermore, the selection of the mother wavelet plays an important role, since track defects appearance and vibration patterns strongly depend on this parameter. In order to show this, a parallel wavelet analysis has been carried out. Results of Fig. 4 are shown in Fig. 11 for a Morlet wavelet and a number of scales of 500, which yield the best results in terms of pattern identification. For this representation, the frequency associated to each scale level has been adopted in the $y$-axis. Frequency is represented in a logarithmic scale, as this is the natural way wavelets work.

Comparison between Figs. 4 and 11 shows that short track defects and singularities appear with better time resolution in the WT than in the STFT. In opposition, vibration modes show poorer resolution. This frequency resolution worsens as frequency increases. Indeed, whereas $f_{\text {pe }}$ (parametric excitation) and $f_{\mathrm{s}}$ (sleeper on ballast vibration) can be properly identified, higher vibration modes such as $f_{\mathrm{r}}$ (rail on railpad vibration) or $f_{\mathrm{pp}}$ (pin-pin resonance) cannot be distinguished.

This fact is also supported by Fig. 12, which shows the WT of the acceleration signal of Fig. 3a. Whereas in Fig. 3a, vibrations induced by the motors clearly appear on the 
spectrogram, such vibrations cannot be identified in Fig. 12, since there is not enough frequency resolution at these frequency levels.

\section{Conclusions}

A surveying approach capable of detecting and classifying the different track singularities and vibration modes has been presented. This detection and classification is achieved in both time and frequency domains. For this purpose, several run tests have been carried out on Metrovalencia tracks involving the analysis of axlebox accelerations. It is shown how with a time-frequency representation based on the conventional STFT, by choosing the appropriate values for the different parameters, an acceptable resolution in both time and frequency domain is achieved. This allows locating and distinguishing some track vibrations modes and singularities. The research further shows how the STFT representation even improves some current analyses performed with WT.

Therefore, the findings of this study are:

- For speeds lower than $80 \mathrm{~km} / \mathrm{h}$ and track configurations shown in Table 4, the monitoring of axlebox accelerations at a sampling frequency of $2.5 \mathrm{kHz}$ with an anti-aliasing filter of $1 \mathrm{kHz}$ allows analysing the most relevant track frequencies in terms of maintenance purposes.

- The location of axlebox accelerators on a motor wheelset hinders the analysis of track vibrations because a wide range of motor-induced vibrations are overlapped. Axlebox accelerators placed on trailer wheelsets provide cleaner spectrograms.

- A Hamming time window with a time length between $0.3 \mathrm{~s}$ and $0.5 \mathrm{~s}$ with a $95 \%$ overlapping has been found to be the most suitable time window for the spectrogram in order to better appreciate the different track defects and vibration modes.

- A Wavelet analysis does not properly show vibration modes with frequency higher than $100 \mathrm{~Hz}$, though detection of track singularities may be improved with respect to the spectrogram. This is affected by various parameters, such as the choice of mother wavelet, the resolution level, etc.

- With these parameters, some track singularities and defects, such as welded and glued joints, turnout frogs and squats, together with the main track vibration modes can be properly featured and classified, avoiding the use of parallel analysis tools such as time-domain peaks analysis, conventional Discrete Fourier Transforms, etc.

\section{Acknowledgements}

The authors would like to thank Metrovalencia and its staff for their collaboration and support provided during the tests.

\section{Funding}

Project Funded by Geoconcept Co. Ltd. and the Spanish Ministry of Economy and Competitiveness. Ref. IDI-20110461.

\section{References}


[1] Grassie, S. L., 1996, "Measurement of railhead longitudinal profiles: a comparison of different techniques," Wear, 191(1-2), pp. 245-251.

[2] Grassie, S. L., 1996, "Short wavelength rail corrugation: field trials and measuring technology," Wear, 191(1-2), pp. 149-160.

[3] Grassie, S. L., Saxon, M. J., and Smith, J. D., 1999, "MEASUREMENT OF LONGITUDINAL RAIL IRREGULARITIES AND CRITERIA FOR ACCEPTABLE GRINDING," Journal of Sound and Vibration, 227(5), pp. 949-964.

[4] Insa, R., Inarejos, J., Salvador, P., and Baeza, L., 2012, "On the filtering effects of the chord offset method for monitoring track geometry," Proceedings of the Institution of Mechanical Engineers. Part F. Journal of Rail and Rapid Transit, 226(6), p. 5.

[5] Ward, C. P., Weston, P. F., Stewart, E. J. C., Li, H., Goodall, R. M., Roberts, C., Mei, T. X., Charles, G., and Dixon, R., 2011, "Condition Monitoring Opportunities Using Vehicle-Based Sensors," Proceedings of the Institution of Mechanical Engineers, Part F: Journal of Rail and Rapid Transit, 225(2), pp. 202-218.

[6] Kouroussis, G., Caucheteur, C., Kinet, D., Alexandrou, G., Verlinden, O., and Moeyaert, V., 2015, "Review of Trackside Monitoring Solutions: From Strain Gages to Optical Fibre Sensors," Sensors, 15, p. 25.

[7] Weston, P., Roberts, C., Yeo, G., and Stewart, E., 2015, "Perspectives on railway track geometry condition monitoring from in-service railway vehicles," Vehicle System Dynamics, 53(7), pp. 1063-1091.

[8] Andrade, A. R., and Teixeira, P. F., 2013, "Hierarchical Bayesian modelling of rail track geometry degradation " Proceedings of the Institution of Mechanical Engineers. Part F: Journal of Rail and Rapid Transit, 227(4), p. 12.

[9] Andrade, A. R., and Teixeira, P. F., 2015, "Statistical modelling of railway track geometry degradation using Hierarchical Bayesian models," Reliability Engineering \& System Safety, 142, pp. 169-183.

[10] UIC, 2009, "Testing And Approval Of Railway Vehicles From The Point Of View Of Their Dynamic Behaviour - Safety - Track Fatigue - Ride Quality," UIC 518.

[11] Alfi, S., Bionda, S., Bruni, S., and Gasparetto, L., "Condition monitoring of suspension components in railway bogies," Proc. Railway Condition Monitoring and Non-Destructive Testing (RCM 2011), 5th IET Conference on, pp. 1-6.

[12] Li, Z., Molodova, M., Zhao, X., and Dollevoet, R., 2010, "Squat Treatment by Way of Minimum Action Based on Early Detection to Reduce Life Cycle Costs," Proceedings of the 2010 Joint Rail Conference, ASME, ed., ASME, Urbana, IL, USA.

[13] Li, Z., Dollevoet, R., Molodova, M., and Zhao, X., 2011, "Squat growth-Some observations and the validation of numerical predictions," Wear, 271(1-2), pp. 148-157. [14] Li, Z., Zhao, X., Esveld, C., Dollevoet, R., and Molodova, M., 2008, "An investigation into the causes of squats-Correlation analysis and numerical modeling," Wear, 265(9-10), pp. 1349-1355.

[15] Molodova, M., Zili, L., Nunez, A., and Dollevoet, R., 2014, "Automatic Detection of Squats in Railway Infrastructure," Intelligent Transportation Systems, IEEE Transactions on, 15(5), pp. 1980-1990.

[16] Zili, L., Molodova, M., Nunez, A., and Dollevoet, R., 2015, "Improvements in Axle Box Acceleration Measurements for the Detection of Light Squats in Railway Infrastructure," Industrial Electronics, IEEE Transactions on, 62(7), pp. 4385-4397.

[17] Li, Z., Oregui, M., Carroll, R., Li, S., and Moraal, J., 2012, "Detection of Bolt Tightness of Fish-plated Joints using Axle Box Acceleration," Proceedings of the First International Conference on Railway Technology: Research, Development and Maintenance, J. Pombo, ed., Civil-Comp Press, Las Palmas de Gran Canaria, Spain. 
[18] Oregui, M., Li, Z., and Dollevoet, R., 2015, "Identification of characteristic frequencies of damaged railway tracks using field hammer test measurements," Mechanical Systems and Signal Processing, 54-55, pp. 224-242.

[19] Popović, Z., Lazarević, L., Brajović, L., and Vilotijević, M., 2015, "The Importance of Rail Inspections in the Urban Area -Aspect of Head Checking Rail Defects," Procedia Engineering, 117, pp. 601-613.

[20] Molodova, M., Li, Z., Núñez, A., and Dollevoet, R., 2014, "Validation of a finite element model for axle box acceleration at squats in the high frequency range," Computers \& Structures, 141, pp. 84-93.

[21] Grassie, S. L., 2005, "Rail corrugation: advances in measurement, understanding and treatment," Wear, 258(7-8), pp. 1224-1234.

[22] Bocciolone, M., Caprioli, A., Cigada, A., and Collina, A., 2007, "A measurement system for quick rail inspection and effective track maintenance strategy," Mechanical Systems and Signal Processing, 21(3), pp. 1242-1254.

[23] Torstensson, P. T., and Schilke, M., 2013, "Rail corrugation growth on small radius curves-Measurements and validation of a numerical prediction model," Wear, 303(12), pp. 381-396.

[24] Hubbard, P., Ward, C., Dixon, R., and Goodall, R., 2013, "Real time detection of low adhesion in the wheel/rail contact," Proceedings of the Institution of Mechanical Engineers, Part F: Journal of Rail and Rapid Transit, 227(6), pp. 623-634.

[25] Coudert, F., Sunaga, Y., and Takegami, K., 1999, "Use of Axle Box Acceleration to Detect Track and Rail Irregularities," WCRR, p. 7.

[26] Tsunashima, H., Nagamuna, Y., Matsumoto, A., Mizuma, T., and Mori, H., 2012, "Condition Monitoring of Railway Track Using In-Service Vehicle," Reliability and Safety in Railway, X. Perpinya, ed., In Tech, Rijeka, Croatia, pp. 333-356.

[27] Naganuma, Y., Kobayashi, M., Nakgawa, M., and Okumura, T., 2008, "Condition Monitoring of Shinkansen Tracks using Commercial Trains," 4th IET International Conference on Railway Condition Monitoring, T. I. o. E. a. T. R. Network, ed., IET Conference Publications, Derby, UK, p. 6.

[28] Cantero, D., and Basu, B., 2014, "Railway infrastructure damage detection using wavelet transformed acceleration response of traversing vehicle," Struct. Control Health Monit., Article in press.

[29] Bruni, S., Anastasopoulos, I., Alfi, S., Van Leuven, A., and Gazetas, G., 2009, "Effects of train impacts on urban turnouts: Modelling and validation through measurements," Journal of Sound and Vibration, 324(3-5), pp. 666-689.

[30] Hory, C., Bouillaut, L., and Aknin, P., 2012, "Time-frequency characterization of rail corrugation under a combined auto-regressive and matched filter scheme," Mechanical Systems and Signal Processing, 29(0), pp. 174-186.

[31] Molodova, M., Li, Z., and Dollevoet, R., 2011, "Axle box acceleration: Measurement and simulation for detection of short track defects," Wear, 271(1-2), pp. 349-356.

[32] Caprioli, A., Cigada, A., and Raveglia, D., 2007, "Rail inspection in track maintenance: A benchmark between the wavelet approach and the more conventional Fourier analysis," Mechanical Systems and Signal Processing, 21(2), pp. 631-652.

[33] Schenkendorf, R., and Groos, J., 2015, "Global Sensitivity Analysis applied to Model Inversion Problems: A Contribution to Rail Condition Monitoring," International Journal of Prognostics and Health Management 6^.Sp4, p. 14.

[34] Tsai, H.-C., Wang, C.-Y., Huang, N. E., Kuo, T.-W., and Chieng, W.-H., 2015, "Railway track inspection based on the vibration response to a scheduled train and the 
Hilbert-Huang transform," Proceedings of the Institution of Mechanical Engineers, Part F: Journal of Rail and Rapid Transit, 229(7), pp. 815-829.

[35] Kalker, J. J., and Grassie, S. L., 1990, "Wheel-rail rolling contact theory," Mechanics and Fatigue in Wheel/Rail Contact, Elsevier, Oxford, pp. 243-261.

[36] Kalker, J. J., 1991, "Wheel-rail rolling contact theory," Wear, 144(1-2), pp. 243261.

[37] De Man, A. P., 2000, "Pin-pin resonance as a reference in determining ballasted railway track vibration behaviour," Heron, 45(1), p. 17.

[38] Pombo, J., Ambrósio, J., Pereira, M., Verardi, R., Ariaudo, C., and Kuka, N., 2011, "Influence of track conditions and wheel wear state on the loads imposed on the infrastructure by railway vehicles," Computers \& Structures, 89(21-22), pp. 1882-1894. [39] Boashash, B., 1992, Time-frequency signal analysis : methods and applications, Longman Cheshire [etc.], Melbourne [etc.].

[40] Grassie, S. L., 2012, "Rail irregularities, corrugation and acoustic roughness: characteristics, significance and effects of reprofiling," Proceedings of the Institution of Mechanical Engineers Part F-Journal of Rail and Rapid Transit, 226(F5).

[41] Steenbergen, M. J. M. M., "Physics of railroad degradation: The role of a varying dynamic stiffness and transition radiation processes," Computers \& Structures(0).

[42] Varandas, J. N., Hölscher, P., and Silva, M. A. G., 2011, "Dynamic behaviour of railway tracks on transitions zones," Computers \& Structures, 89(13-14), pp. 14681479.

[43] Insa, R., Salvador, P., Inarejos, J., and Roda, A., 2012, "Analysis of the influence of under sleeper pads on the railway vehicle/track dynamic interaction in transition zones," Proceedings of the Institution of Mechanical Engineers. Part F: Journal of Rail and Rapid Transit, 226(4), p. 12. 


\section{Figure captions}

1_ Fig. 1. Main train and track components, their representation in a conceptual train/track dynamic interaction model and location of accelerometers.

2_ Fig. 2. Spectrogram showing the same accelerations sampled at $15 \mathrm{kHz}$ (a) and at 2.5 $\mathrm{kHz}$ (b). Speed $75-30 \mathrm{~km} / \mathrm{h}$.

3_ Fig. 3. Spectrogram corresponding to the same track stretch surveyed from a motor wheelset (a) and from a trailer wheelset (b). Speed $10-30 \mathrm{~km} / \mathrm{h}$

4_ Fig. 4. Spectrogram showing different track singularities. Speed $35 \mathrm{~km} / \mathrm{h}$

5_Fig. 5. Welded Joint

6_ Fig. 6. Glued joint

7_ Fig. 7. Squat at the nose of a turnout frog

8_ Fig. 8. Example of turnout frog

9_ Fig. 9. Zoom to the $0-100 \mathrm{~Hz}$ frequency band for the same spectrogram shown in Fig. 4

10_ Fig. 10. Spectrogram showing different resonances, vibration modes and track configurations. Speed $40 \mathrm{~km} / \mathrm{h}$

11_ Fig. 11. Wavelet transform corresponding to the spectrogram of Fig. 4.

12_ Fig. 12. Wavelet transform corresponding to the spectrogram of Fig. 3a. 


\section{Table captions}

1_ Table 1. Classification of track vertical defects upon their wavelengths. Source: Adif (internal document)

2_ Table 2. Excitation frequencies of different wavelengths when travelling at different speeds and its possible coupling to the different train and track elements:

$\checkmark$ Track elements; $\square$ Unsprung masses; $\square$ Semi-sprung masses; $\square$ Sprung masses

2_ (Black and white) Table 2. Excitation frequencies of different wavelengths when travelling at different speeds and its possible coupling to the different train and track elements:

๑Track elements; $\quad$ पUnsprung masses; $\square$ Semi-sprung masses; $\square$ Sprung masses

3_ Table 3. Adif classification of track defects upon the axlebox vertical accelerations. Source: Adif

4_ Table 4. Different features for both track configurations.

5_ Table 5. Specific features of the different run tests showing the sampling frequency and the anti-aliasing filtering choice.

6_ Table 6. Observed appearances for different window lengths 